\title{
HIOP-Reader: Automated Data Extraction for the Analysis of Manually Recorded Nycthemeral IOPs and Glaucoma Progression
}

Running Title: Automated 24h IOP Analysis and Glaucoma Agorastou, V. ${ }^{1}$; Schön, J. ${ }^{2}$; Verma-Fuehring, R. ${ }^{1}$; Dakroub, M. ${ }^{1}$; Hillenkamp, J. ${ }^{1}$

Puppe, F. ${ }^{2}$; Loewen, N.A. ${ }^{1}$

1: Department of Ophthalmology, University of Würzburg, Würzburg, Germany

2: Institute for Artificial Intelligence and Knowledge Systems, Department of Informatics, University of Würzburg, Würzburg, Germany

Nils A. Loewen, MD, PhD.

Department of Ophthalmology

University of Würzburg, Josef-Schneider-Straße 11,

97080 Würzburg, Germany.

Email: loewen.nils@gmail.com

Conflict of Interest: None.

Précis: Nycthemeral intraocular pressure (IOP) monitoring is commonly used in Europe to detect glaucomatous IOP values. Using efficient, machine-learning data extraction tools to study manually drawn IOP charts, we found no correlation between any IOP parameters and glaucoma progression.

\section{Keywords:}

glaucoma progression; nycthemeral intraocular pressure; mean ocular perfusion pressure 


\begin{abstract}
Purpose: Nycthemeral (24-hour) glaucoma inpatient intraocular pressure (IOP) monitoring has been used in Europe for more than 100 years to detect peaks missed during regular office hours. Data supporting this practice is lacking, partially because it is difficult to correlate manually drawn IOP curves to objective glaucoma progression. To address this, we deployed automated IOP data extraction tools and tested for a correlation to a progressive retinal nerve fiber layer loss on spectral-domain optical coherence tomography (SDOCT).

Methods: We created and deployed a machine-learning image analysis software to extract IOP data from hand-drawn, nycthemeral IOP curves of 225 retrospectively identified glaucoma patients. The relationship between demographic parameters, IOP and mean ocular perfusion pressure (MOPP) data to SDOCT data was analyzed. Sensitivities and specificities for the historical cut-off values of $15 \mathrm{mmHg}$ and $22 \mathrm{mmHg}$ in detecting glaucoma progression were calculated.

Results: IOP data could be extracted efficiently. The IOP average was $15.2 \pm 4.0 \mathrm{mmHg}$, nycthemeral IOP variation was $6.9 \pm 4.2 \mathrm{mmHg}$, and MOPP was $59.1 \pm 8.9 \mathrm{mmHg}$. Peak IOP occurred at $10 \mathrm{AM}$ and trough at 9 PM. Disease progression occurred mainly in the temporal-superior and -inferior SDOCT sectors. No correlation could be established between demographic, IOP, or MOPP parameters and SDOCT disease progression. The sensitivity and specificity of both cut-off points (15 and $22 \mathrm{mmHg}$ ) were insufficient to be clinically useful. Outpatient IOPs were non-inferior to nycthemeral IOPs.
\end{abstract}

Conclusion: IOP data obtained during a single visit make for a poor diagnostic tool, no matter whether obtained using nycthemeral measurements or during outpatient hours. 


\section{Introduction}

The need for better diagnostic options in glaucoma is critical, as this disease only presents symptoms at an advanced stage and is often diagnosed late. ${ }^{1} 42 \%$ of all primary open-angle glaucoma (POAG) patients ultimately go blind in one eye,$^{2}$ partially because of this. To better assess the effectiveness of the treatment and to detect pressure peaks that are not recognized during office hours, ${ }^{3}$ patients in German-speaking countries are often admitted for nycthemeral (24-hour) intraocular pressure (IOP) profiles. ${ }^{4}$ Such monitoring generates costs averaging EUR 643 per night ${ }^{5,6}$ and has been obtained, based on verbally communicated past use patterns at many clinics, at least approximately one million times in the last 100 years ${ }^{4,7-9}$ to aid in the diagnosis and treatment of glaucoma. However, evidence supporting 24-hour IOP profiles for identifying IOPs above target or larger than normal IOP fluctuations $s^{4,8-11}$ is at most expert opinion (level V) ${ }^{12-14}$. The absence of strong evidence for 24-hour IOP profiles as a diagnostic tool in glaucoma is surprising, considering the contrast to the high-quality level I evidence that establishes IOP as the preeminent cause of glaucoma ${ }^{12-14}$. Damage from high IOP is an experimentally demonstrated pathomechanism of glaucoma in nonhuman primates. ${ }^{15,16}$ Elevated IOP levels are strongly correlated to human glaucoma incidence ${ }^{17,18}$, and their treatment reduces glaucoma onset and progression. ${ }^{19,20}$ Moreover, IOP fluctuations and pressure peaks during outpatient clinic hours have previously been associated with glaucoma progression. ${ }^{21}$

One reason for the missing link between vast historical records of 24-hour IOP profiles and glaucoma progression may be the difficulty in extracting data from manually drawn IOP curves that are paper-based and correlating them to objective, statistically significant progression. To address this, we created a computer-aided image analysis of 24-hour IOP profiles. We matched them to worsening retinal nerve fiber layer thickness using current spectral-domain optical coherence tomography and software (SPECTRALIS SD-OCT, Heidelberg Engineering, Heidelberg, Germany). Similarly, we estimated the ocular perfusion pressure and determined the strength of correlation to progression.

High IOP damages the axons of retinal ganglion cells primarily at the level of the lamina cribrosa, a biomechanical weak point. ${ }^{22,23}$ Too low an ocular perfusion pressure ${ }^{24}$ is considered to be a secondary contributing factor. Based on this, our primary hypothesis was that 24-hour inpatient IOPs are correlated to a statistically significant decline of the retinal nerve fiber layer (RNFL), in particular the temporal-superior, temporal or temporal-inferior RNFL. Our secondary hypothesis was that ocular perfusion pressure is correlated to glaucoma progression. 


\section{Methods}

\section{Study design}

This retrospective chart review was carried out at the Department of Ophthalmology of the University of Würzburg. It abided by the principles stated in the declaration of Helsinki. Due to its retrospective nature, informed consent was waived by the Institutional Review Board of the University of Würzburg. Charts of 225 patients admitted to the ophthalmology inpatient unit at the University Hospital of Würzburg for nycthemeral IOP monitoring from 2017 to 2019 were analyzed to comprise two years since the introduction of OCT-aided progression analysis in this hospital. Only right eyes were analyzed to reduce bias. Patients included had a diagnosis of primary open-angle glaucoma (POAG), low-tension glaucoma (LTG), pseudoexfoliation glaucoma (PXG), pigmentary glaucoma (PG), and juvenile glaucoma (JOAG). Patients with terminal, neovascular, uveitic, or angle-closure glaucoma were excluded from the study. Terminal glaucoma was defined as having a nearly complete visual field loss or a cup-to-disc ratio of 1.0 .

Parameters recorded included age, gender, diagnosis, history of surgery, family history of glaucoma, medications, slit lamp, fundoscopic examination findings, and the central corneal thickness. The 24-hour IOP protocol established in this hospital called for measurements in the habitual position with 10 AM, 2 PM, 5 PM, and 9 PM readings obtained by Goldmann applanation tonometry (HaagStreit, Köniz, Switzerland) in the sitting position, and the 12 AM measurement obtained by Perkins applanation tonometry (Perkins MK3, Haag-Streit, Köniz, Switzerland) in the supine position. IOPs were recorded on paper charts using blue for right eyes and red for left eyes (Fig. 1). Each subject's 24-hour IOP data was fit to a cosine curve. Because there were only five measurements, instead of at least twelve, this fit was done manually using a sparkline macro ${ }^{3,25}$. The acrophase was estimated by defining it as the phase timing, in which a peak IOP during the 24 hours was reached. Paper-based 24hour IOP profiles were examined using a custom-made computer-aided image analysis program. Values noted were: $T_{\max }, T_{\min }, T_{a v g}$, and IOP $P_{v a r}\left(T_{\max }-T_{\min }\right)$. Additionally, the mean ocular perfusion pressure (MOPP) was calculated as two-thirds of the difference between the mean arterial pressure and $\mathrm{T}_{\text {avg. }}$.

\section{Image analysis of manually recorded 24-hour IOP profiles}

We wrote the Python-based program HIOP-Reader ${ }^{26}$ to extract patient name, examination date, and the IOP values on the $y$-axis with their corresponding time on the $x$-axis. We used OpenCV ${ }^{27}$ for image 
processing, Tesseract ${ }^{28}$ for optical character recognition, and TensorFlow ${ }^{29}$ and scikit-learn ${ }^{30}$ for machine learning. The image analysis was divided into three parts: preprocessing, value detection, and name and date extraction.

The main goal of preprocessing was to detect the frame containing the IOP profile and crop the image to it. We achieved this by searching for curves, joining all continuous points with the same intensities. In OpenCV, this is referred to as contours. To improve the accuracy of finding contours, we binarized the image by applying adaptive thresholding. We used Gaussian adaptive thresholding, which calculates the Gaussian weighted sum over a neighborhood of, in our case, $27 \times 27$ pixels, to find an appropriate threshold value. This threshold, minus a constant $C=10$, was then used to binarize the image. From the binary image, we chose the largest resulting contour as the main frame of the image. To make the process more robust, we ensured that the resulting contour is a rectangle. This was done by approximating the contour using the Douglas-Peucker algorithm, ${ }^{31,32}$ ensuring that the contour consisted of four lines even when the frame was cut-off or other artifacts were obstructing the frame. Next, we checked the angles between the four lines of the approximated contour, ensuring that it was at least close to a rectangle. Finally, we cropped the image to the resulting approximation of the largest contour, resulting in an image cropped to the main frame of the IOP profile. After cropping, all scanned images had the same format and size, enabling us to do precise pixel position-based operations.

To extract the IOP values entered into the profile, we detected the lines representing the different examination times using the Canny edge detection algorithm ${ }^{33}$ and Hough line transformation. ${ }^{34}$ Any falsely detected or horizontal lines were removed. This left us with the precise positions of the lines representing different times. For each line, a neighborhood around it was considered when searching for IOP values. We exploited the fact that all IOP values for the left eye were entered in red, while all values for the right eye were entered in blue and created color-specific masks. These masks only contained the part of the image that was blue or red, respectively. IOP values were collected using these masks and the immediate vicinity of each line. Lastly, since all images had the same format, the IOP value could be directly inferred from the pixel position of the detected entry.

To capture the date of the 24-hour IOP profile, we applied a traditional machine learning approach. First, we isolated the area where the date was recorded and separated the numbers and the delimiters using contours. The numbers were then predicted using a convolutional neural network trained on the Modified National Institute of Standards and Technology (MNIST) dataset. ${ }^{35}$ As the 
patient names were mostly recorded using machine-written labels, optical character recognition with Tesseract ${ }^{28}$ could be used to extract all machine-written text on the form. We used regular expressions on the extracted text to find patient names. All information was manually confirmed and stored as CSV files. To allow for rapid editing and error correction, we developed a graphical user interface for the program.

\section{Statistical Analysis}

\section{Data Management}

Confirmatory and exploratory data analysis was performed using JMP (JMP 15.2.1, SAS Institute Inc., Cary, North Carolina, USA). Means along with standard deviations were calculated for continuous variables, while percentages were computed for categorical variables. A Kolmogorov-Smirnov test was run to assess continuous variables for a normal distribution. Bivariate analysis was used to study the relationship between various IOP parameters. Independent sample t-tests were used to compare means of continuous variables, whereas a chi-square test compared those of categorical variables. Spearman's rank-order correlation coefficient (rather than a Pearson's correlation coefficient) was reported if data sets were not normally distributed. For all our analyses, a p-value of 0.05 or less was considered statistically significant.

\section{OCT \& Disease Progression Analysis}

Disease progression was assessed using a Spectral Domain OCT (SPECTRALIS OCT, Heidelberg Engineering $\mathrm{GmbH}$, Heidelberg, Germany). The retinal nerve fiber layer (RNFL) thickness (in micrometers) of all peripapillary sectors was recorded. Changes in RNFL thickness were evaluated using commercial software (HEYEX Version 2.4.1., Heidelberg Engineering GmbH, Heidelberg, Germany), which provided both the rate of RNFL loss and a statistical comparison to a normal age-related RNFL loss rate. In this way, progression was calculated both as a continuous and a dichotomous variable. Linear regression was utilized to assess the relationship between several continuous variables (such as $\mathrm{IOP}_{\mathrm{var}}$ ) and the rate of RNFL loss, representing disease progression. A contingency analysis was carried out to determine the sensitivity and specificity of using 15 and $22 \mathrm{mmHg}$ as $\mathrm{T}_{\max }$ cut-off points in detecting disease progression in any sector. These sensitivity and specificity measurements were then calculated with 10 AM, 2 PM, and 5 PM values to compare these values to a hypothetical outpatient situation. 


\section{Results}

Table 1 depicts the demographic variables of the 225 patients included in this analysis. Five eyes were excluded due to meeting our criteria for terminal glaucoma. There were 137 women (61\%) and 88 men (39\%). Women were significantly older than men (77.0 \pm 10.0 years versus $72.8 \pm 12.6$ years, respectively, $p=0.006)$. The diagnoses included were POAG $(n=130,57.8 \%)$, LTG $(n=41,18.2 \%)$, PXG $(n=39,17.3 \%)$, GS $(n=8,3.6 \%)$, PG $(n=4,1.8 \%)$, and JOAG ( $n=3,1.3 \%)$. Patients with POAG, LTG, and PXG were older than those with PG and JOAG ( $p<0.001)$ (Fig. 2). Compared to the 3:2 ratio of women to men in this study, there were disproportionately more women $(78 \%, n=32)$ with LTG than men $(22 \%, n=9)$. There was no statistically significant difference in the number of medications per eye in both groups, with an average of 2.2 drops in each group ( $p=1.0$, Table 1). Fifty-eight patients had four different topical glaucoma medications, with prostaglandin analogs being the most prescribed medication (31.6\%), followed by carbonic anhydrase inhibitors (27.0\%), alpha agonists (22.0\%), and beta-blockers (19.4\%). The mean central corneal thickness (CCT) was $526.3 \pm 35.7 \mu \mathrm{m}$. There was no gender difference (females: $538.6 \pm 34.0 \mu \mathrm{m}$, males: $534.838 .3 \mu \mathrm{m}$, respectively, $\mathrm{p}=0.43$ ).

We evaluated HIOP-Reader on 100 IOP profiles. An average of 3.5977 seconds was needed to process a file, not accounting for human error correction. The IOP curves showed a mean of 8.4266 entries per eye. The names were detected correctly with an accuracy of $75.32 \%$, the detection of the date was only accurate in $42.85 \%$ of the cases. The entered values were detected with high accuracy. On average, there were 0.4675 falsely detected entries per IOP curve. An average of 0.3376 entries per IOP curve was not detected. For the detected entries, the average distance between the actual value and the predicted value was 0.0927 . The evaluation was performed on standard consumer hardware from 2019 with a 2,4 GHz Quad-Core Intel Core i5-8279U CPU and 16 GB of random access memory. LTG had a significantly lower $T_{\text {avg }}$ and $T_{\max }$ than POAG and PXG ( $p=0.005$ and $p<0.001$, respectively; Fig. 3). The CCT of LTG was not significantly different from POAG or PXG (both $p>0.05$ ). IOP $_{\text {var }}$ was correlated with $\mathrm{T}_{\max }$ (correlation 0.8, $\mathrm{p}<0.001$ ) and with $\mathrm{T}_{\text {avg }}$ (correlation 0.3, $\mathrm{p}<0.001$ ) but not with $\mathrm{T}_{\min }$.

The observed average IOPs were relatively similar throughout the day and ranged from a peak of $15.8 \pm 5.1 \mathrm{mmHg}$ at 10:00 to a trough of $14.5 \pm 4.6 \mathrm{mmHg}$ at 21:00 ( $\mathrm{p}=0.519 ;$ Fig. 4). One hundred-nine patients had an acrophase with peak IOP at 10:00 AM. The acrophase spread was $8.4 \pm 3.8$ hours. When all 24-hour IOP curves were adjusted to have matching acrophases, a peak IOP of $18.1 \pm 5.3 \mathrm{mmHg}$ was reached at 10:00 AM and a trough of 14.2 $\pm 4.1 \mathrm{mmHg}$ at 21:00 ( $p<0.001 ;$ Fig. 5). 
OCT progression data were available in 116 out of 225 patients. Of those, $42 \%$ were progressors with a significantly worsening retinal nerve fiber layer thickness. More patients had progression in the TI (31\%) and TS (36\%) sector than in T (22\%). Most progressions occurred in the TS and TI sectors (Fig. 6). Between progressors and non-progressors, there were no differences in age, gender, or type of glaucoma, nor was there a difference in their IOP peak time, IOP $\mathrm{var}_{\text {, }} \mathrm{T}_{\max }, \mathrm{T}_{\mathrm{avg}}$, or $\mathrm{T}_{\min }($ all $\mathrm{p}>0.05)$. IOPvar was $6.3 \pm 3.6 \mathrm{mmHg}$ in progressors and $6.8 \pm 3.9 \mathrm{mmHg}$ in non-progressors, respectively. There was no difference in age. The RNFL decline in these progressors had an average of $2.3 \pm 1.7$ microns per year. Applying an old concept that IOP variations of more than $5 \mathrm{mmHg}$ may indicate glaucoma progression underlying the rationale of obtaining inpatient, 24-hour IOP measurements ${ }^{4}$, sensitivity for such variation to detect glaucoma progression was $68 \%$ and specificity $25 \%$.

Applying a historical cutoff of $22 \mathrm{mmHg}$ as an IOP considered too high, sensitivity was only $7 \%$, and specificity was $87 \%$. When a cutoff of $15 \mathrm{mmHg}$ was chosen, corresponding to a normal IOP of healthy eyes often viewed as suboptimal for moderate to advanced glaucoma, sensitivity was $69 \%$, and specificity was $23 \%$. Table 2 shows the sensitivity and specificity of those cut-off values obtained during 24-hour measurements and compares them to the same IOP criteria if those were applied to regular outpatient clinic hours. The specificity of the criteria "15 mmHg" during outpatient hours was slightly better than when applied to inpatient 24-hour measurements, while the criteria "22 mmHg" were very similar. Figure 7 applies the concept of Tmax and Tavg as a test for glaucoma progression to a receiver operating characteristic $(\mathrm{ROC})$ curve. All curves, regardless of inpatient or outpatient values, were close to the reference line, indicating poor performance.

Table 3 summarizes the correlations we found. $T_{\max }, T_{a v g}, T_{\min }$, and IOPvar were not correlated to the slope (speed) of RNFL loss ( $p>0.05$ ). These parameters were also not correlated to structural differences between the expected, normative RNFL thickness or the actual (absolute) RNFL thickness measured by the SPECTRALIS OCT.

The estimated MOPP was $59.1 \pm 8.9 \mathrm{mmHg}$. This parameter did not differ by glaucoma type $(p=0.42)$ or sex $(p=0.79)$. MOPP correlated negatively and weakly to the slope of the temporal superior retinal fiber layer thickness $(r=-0.09, p=0.04)$, to $T_{\text {avg }}(r=-0.14, p=0.04), T_{\max }(r=-0.15, p=0.03)$ and $T_{\min }$ $(r=-0.14, p=0.04)$ but not to IOPvar $(p=0.72)$. There was no significant correlation between MOPP and worsening glaucoma $(p=0.34)$. This was also not the case in LTG $(p=0.14)$. 


\section{Discussion}

We developed a high-efficiency reader specifically to extract nycthemeral IOP data from manually drawn charts and assessed disease progression using an SD-OCT with progression analysis software. We found no significant relationship between nycthemeral IOP measurements and glaucoma progression, despite the best efforts.

HIOP-Reader allowed us to rapidly process and extract a large amount of image data with a low error rate. This program is made available to the scientific community via GitHub, ${ }^{26,36}$ a public software repository. Further improvements could be made with date extraction using component labeling and support vector machine classification ${ }^{37}$ or Hidden Markov Model ${ }^{38}$ based methods. The functionality that allows for statistical analysis of handwritten IOP profiles worked well. In particular, the program showed resilience to imperfections inherent to IOP profiles drawn manually by different users, and the IOP values were detected with high accuracy. This allowed us to process and use large amounts of handwritten data that would have been hard to acquire. We believe HIOP-Reader will be a useful mining tool to process the many decades of data available at inpatient-based ophthalmology clinics that have performed nycthemeral IOP measurements in the past.

Regarding patient demographics in our study, the gender ratio of women (61\%) to men (39\%) was very similar, almost down to the digit, to that of global glaucoma studies. ${ }^{39,40}$ Among glaucoma subtypes, LTG, in particular, is more prevalent in women, ${ }^{41}$ a pattern seen in our study as well. Except for age, the demographic variables of men and women did not differ.

The idea behind collecting 24-hour IOPs appears to have been that glaucoma patients might have a higher nocturnal peak and a larger IOP variation than normal eyes ${ }^{4,7-9,11}$ when in fact, it has been known for a while that healthy eyes have a larger IOP variation than glaucomatous eyes. ${ }^{42}$ Looking for nocturnal peaks may also be of limited diagnostic value, as an elevated nocturnal IOP in the supine position is a physiological reaction in healthy and glaucomatous eyes. ${ }^{42}$ Research into the relationship between IOP variation and glaucoma progression has produced discordant findings, however. ${ }^{43-47}$ A study of 105 POAG eyes with normal in-office IOP values showed IOP ranges over five days to be an independent risk factor for disease progression (defined as visual field loss). ${ }^{43}$ Similarly, some studies showed short-term (48-hour) and long-term IOP fluctuations to be correlated to visual field progression. ${ }^{44,47,48}$ Other investigators failed to corroborate these factors, however. ${ }^{45,46}$ One reason for this may be the inclusion of glaucoma patients undergoing medical therapy, who have a smaller fluctuation range. ${ }^{49}$ A 2007 study on 71 treated POAG eyes compared office IOP (9 AM - 6 PM) page 9 of 26 
to 24-hour IOP readings and showed no statistical significance in the mean IOPs of both groups. ${ }^{50}$ In another study, the office IOP fluctuation was substantially lower than that of 24-hour measurements, and the two could not be correlated..$^{50}$ Interestingly, a different study found that the mean outpatient IOP could, in fact, be used to predict both mean and peak nycthemeral IOPs. ${ }^{51}$

We found nycthemeral and office IOP variables to have an inadequate sensitivity and specificity in identifying progressors, as the ROC curves demonstrate. Well-performing medical diagnostic tests, as the SDOCT, have a value close to $90 \%$ in both parameters (resulting in a hyperbolic curve shape). ${ }^{52}$ This does not mean that there is no connection between 24-hour IOP variables and glaucoma progression. Instead, our findings highlight the challenges of implementing a well-intended test in a busy clinical environment without the proper methods. New evidence has emerged demonstrating that IOP peaks and variation in 24-hour IOP measurements are indeed linked to glaucoma pathogenesis when operator-independent, implantable IOP-sensors or contact lenses are used to record pressures at home..$^{53-56}$

The retrospective IOP data we processed in this study had considerable shortcomings. Values were recorded with a commitment to seemingly arbitrarily set times, unevenly distributed throughout the day, and at an interval larger than the 2-hour interval of IOP sleep lab studies. ${ }^{3,25}$ Such a customized schedule might fit clinicians' work schedules better, but it prevents finding the best fitting cosine curve and the peak (acrophase) as the phase timing of the 24-hour rhythm. ${ }^{25}$ The IOP peak at 10 AM in our data appeared to be later than in previous studies, but this is unlikely to be the actual phase timing. Other studies reported peaks around 5:30 AM, ${ }^{57} 6 \mathrm{AM}, 588 \mathrm{AM},{ }^{59}$ and troughs at $2 \mathrm{PM}^{59} 5$ $\mathrm{PM},{ }^{44}$ and 9:30 $\mathrm{PM},{ }^{57}$ respectively.

We found MOPP to be negatively correlated to $T_{a v g}, T_{\max }$, and $T_{\min }$. This is not surprising, as one would expect the perfusion pressure to increase somewhat as the IOP decreases. However, this did not correlate to progression, as suggested by other studies that examined POAG, PXG, and LTG. ${ }^{19,60-62}$ A reduced nocturnal ocular perfusion pressure, in particular, has been associated with increased structural damage and visual field deterioration in LTG patients. ${ }^{60,63}$ The blood pressure readings we used for the MOPP estimation were obtained on admission during late morning hours, however.

Our study points to several flaws in rationale and execution when obtaining 24-hour inpatient IOPs may be problematic for several reasons. First, values measured during an inpatient stay are unlikely to reflect values at home due to maximized drop compliance in a clinic environment with close observation and the looming threat of an intervention as a stepped-up treatment for uncontrolled IOP page 10 of 26 
levels. Second, diurnal intraocular pressure patterns are neither sustained nor reproducible. ${ }^{64}$ Because of this, a therapeutic decision should not be based on single measurements. Fourth, if a patient is already known to have a statistically significant decline on SDOCT, a test with high sensitivity and specificity, a nycthemeral IOP profile would not be helpful to argue against advancing therapy. Third, a Perkins applanation tonometer was used to measure supine IOPs. Although Perkins can be as accurate as Goldmann applanation tonometry, ${ }^{65,66}$ it is highly operator-dependent and requires experience, not all on-call residents might have. A pneumatonometer ${ }^{67}$ a widely accepted standard with high accuracy and reproducibility, would be a better technique. ${ }^{42,68}$ Given these problems, answers as to why ordering nycthemeral IOP profiles has survived more than a century might then be found in how it is limited primarily to German-speaking countries that could follow expert literature on that topic in this language. ${ }^{4,7-10,69}$ Another reason might be a healthcare structure that still requires inpatients in ophthalmology as a principal pillar of reimbursements at hospitals ${ }^{70}$ even though it became an outpatient specialty in the late 1980 s internationally. ${ }^{71,72}$

In conclusion, we created a software to extract nycthemeral IOP data from hand-drawn IOP charts automatically. No correlation could be found between any IOP parameters or MOPP and objective glaucoma progression. ROC curves indicated a poor performance of this technique as a diagnostic tool. 


\section{References}

1. Prior M, Francis JJ, Azuara-Blanco A, Anand N, Burr JM, Glaucoma screening Platform Study group. Why do people present late with advanced glaucoma? A qualitative interview study. Br J Ophthalmol. 2013;97(12):1574-1578.

2. Peters $D$, Bengtsson B, Heijl A. Lifetime risk of blindness in open-angle glaucoma. Am J Ophthalmol. 2013;156(4):724-730.

3. Loewen NA, Liu JHK, Weinreb RN. Increased 24-hour variation of human intraocular pressure with short axial length. Invest Ophthalmol Vis Sci. 2010;51(2):933-937.

4. Leydhecker W. Die Tagesdruckkurve. In: Leydhecker W, ed. Die Glaukome in Der Praxis: Ein Leitfaden. Springer Berlin Heidelberg; 1991:69-70.

5. Durchschnittliche Verweildauer im Krankenhaus in Deutschland | Statista. Statista. Accessed March 2, 2020. https://de.statista.com/statistik/daten/studie/2604/umfrage/durchschnittliche-verweildauer-imkrankenhaus-seit-1992/

6. Behandlungskosten im Krankenhaus 2017 weiter gestiegen. Published November 19, 2018. Accessed March 2, 2020. https://www.derprivatpatient.de/infothek/nachrichten/behandlungskosten-im-krankenhaus-2017weiter-gestiegen

7. Köllner H. Über die regelmässigen täglichen Schwankungen des Augendruckes und ihre Ursache. Arch Augenheilk. 1916;81:120.

8. Sallmann L. Über die Tagesdruckkurve und über Belastungsproben als Hilfsmittel in der Glaukomdiagnose. In: XIII. Intern. Ophth. Kongreß Amsterdam. Vol 2. ; 1929:482.

9. Sallmann L, Deutsch A. Die klinische Bedeutung der Tagesdruckkurve und der Belastungsproben bei Glaukom. Albrecht von Graefes Archiv für Ophthalmologie. 1930;124(4):624-651.

10. Göbel K, Rüfer F, Erb C. Physiologie der Kammerwasserproduktion sowie der Tagesdruckschwankungen und deren Bedeutung für das Glaukom. Klinische Monatsblätter für Augenheilkunde. 2011;228(02):104-108.

11. Leydhecker W. Die Tagesdruckkurve. In: Leydhecker W, ed. Glaukom in Der Praxis: Ein Leitfaden. Springer Berlin Heidelberg; 1973:88-89.

12. Morton SC, Hassan Murad M, O'Connor E, et al. Quantitative Synthesis-An Update. Agency for Healthcare Research and Quality (US); 2018.

13. Berkman ND, Lohr KN, Ansari M, et al. Grading the Strength of a Body of Evidence When Assessing Health Care Interventions for the Effective Health Care Program of the Agency for Healthcare Research and Quality: An Update. Agency for Healthcare Research and Quality (US); 2013.

14. Burns PB, Rohrich RJ, Chung KC. The levels of evidence and their role in evidence-based medicine. Plast Reconstr Surg. 2011;128(1):305-310.

15. Nickells RW, Schlamp CL, Li Y, et al. Surgical lowering of elevated intraocular pressure in monkeys prevents progression of glaucomatous disease. Exp Eye Res. 2007;84(4):729-736.

16. Quigley HA, Nickells RW, Kerrigan LA, Pease ME, Thibault DJ, Zack DJ. Retinal ganglion cell death in 
experimental glaucoma and after axotomy occurs by apoptosis. Invest Ophthalmol Vis Sci. 1995;36:774786.

17. Sommer A, Tielsch JM, Katz J, et al. Relationship between intraocular pressure and primary open angle glaucoma among white and black Americans. The Baltimore Eye Survey. Arch Ophthalmol. 1991;109(8):1090-1095.

18. Leske MC, Cristina Leske M. Risk Factors for Open-angle Glaucoma. Archives of Ophthalmology. 1995;113(7):918. doi:10.1001/archopht.1995.01100070092031

19. Leske MC, Heijl A, Hussein M, Bengtsson B, Hyman L, Komaroff E. Factors for glaucoma progression and the effect of treatment: the early manifest glaucoma trial. Arch Ophthalmol. 2003;121:48-56.

20. Kass MA, Heuer DK, Higginbotham EJ, et al. The Ocular Hypertension Treatment Study: a randomized trial determines that topical ocular hypotensive medication delays or prevents the onset of primary open-angle glaucoma. Arch Ophthalmol. 2002;120(6):701-713; discussion 829-830.

21. De Moraes CGV, Juthani VJ, Liebmann JM, et al. Risk factors for visual field progression in treated glaucoma. Arch Ophthalmol. 2011;129(5):562-568.

22. Burgoyne CF, Crawford Downs J. Premise and prediction-how optic nerve head biomechanics underlies the susceptibility and clinical behavior of the aged optic nerve head. J Glaucoma. 2008;17(4):318-328.

23. Brazile BL, Yang B, Waxman S, et al. Lamina Cribrosa Capillaries Straighten as Intraocular Pressure Increases. Invest Ophthalmol Vis Sci. 2020;61(12):2.

24. Li Z, Liu D, Wang N. Correlation Among Intraocular Pressure, Intracranial Pressure, and Blood Pressure. Intraocular and Intracranial Pressure Gradient in Glaucoma. Published online 2019:249-252. doi:10.1007/978-981-13-2137-5_36

25. Nelson W, Tong YL, Lee JK, Halberg F. Methods for cosinor-rhythmometry. Chronobiologia. 1979;6(4):305323.

26. Schön J. HIOP-Reader.; 2021. https://github.com/julschoen/HIOP-Reader

27. BRADSKI, G. The OpenCV library. Dr Dobb's J Software Tools. 2000;25:120-125.

28. Kay A. Tesseract: an open-source optical character recognition engine. Linux J. 2007;2007(159):2.

29. Abadi M, Agarwal A, Barham P, et al. TensorFlow: Large-Scale Machine Learning on Heterogeneous Distributed Systems. arXiv [csDC]. Published online March 14, 2016. http://arxiv.org/abs/1603.04467

30. Pedregosa F, Varoquaux G, Gramfort A, et al. Scikit-learn: Machine learning in Python. the Journal of machine Learning research. 2011;12:2825-2830.

31. Li L, Jiang W. An improved Douglas-Peucker algorithm for fast curve approximation. 2010 3rd International Congress on Image and Signal Processing. Published online 2010. doi:10.1109/cisp.2010.5647972

32. Douglas DH, Peucker TK. ALGORITHMS FOR THE REDUCTION OF THE NUMBER OF POINTS REQUIRED TO REPRESENT A DIGITIZED LINE OR ITS CARICATURE. Cartographica. 1973;10(2):112-122.

33. Canny J. A computational approach to edge detection. IEEE Trans Pattern Anal Mach Intell. 1986;8(6):679698. 
34. Hough PVC. Method and means for recognizing complex patterns. 1962. US patent.

35. LeCun Y, Cortes C, Burges CJ. MNIST handwritten digit database. Published online 2010.

36. Tsitoara M. GitHub Primer. Beginning Git and GitHub. Published online 2020:95-104. doi:10.1007/978-14842-5313-7_8

37. Mandal, Ranju and Roy, Partha Pratim and Pal, Umapada. Date field extraction in handwritten documents. In: Proceedings of the 21st International Conference on Pattern Recognition (ICPR2012). IEEE; 2012:533536.

38. Morita ME, Letelier E, El Yacoubi A, Bortolozzi F, Sabourin R. Recognition of handwritten dates on bank checks using an HMM approach. In: Proceedings 13th Brazilian Symposium on Computer Graphics and Image Processing (Cat. No.PR00878). IEEE Comput. Soc; 2002:113-120.

39. Quigley HA, Broman AT. The number of people with glaucoma worldwide in 2010 and 2020. Br J Ophthalmol. 2006;90(3):262-267.

40. National Center for Health Statistics (US). Health, United States, 2019. National Center for Health Statistics (US); 2021.

41. Drance S, Anderson DR, Schulzer M, Collaborative Normal-Tension Glaucoma Study Group. Risk factors for progression of visual field abnormalities in normal-tension glaucoma. Am J Ophthalmol. 2001;131(6):699708.

42. Doshi AB, Liu JHK, Weinreb RN. Circadian Changes in Intraocular Pressure. In: Grehn F, Stamper R, eds. Glaucoma. Springer Berlin Heidelberg; 2009:23-28.

43. Asrani S, Zeimer R, Wilensky J, Gieser D, Vitale S, Lindenmuth K. Large diurnal fluctuations in intraocular pressure are an independent risk factor in patients with glaucoma. J Glaucoma. 2000;9(2):134-142.

44. Matlach J, Bender S, König J, Binder H, Pfeiffer N, Hoffmann EM. Investigation of intraocular pressure fluctuation as a risk factor of glaucoma progression. Clin Ophthalmol. 2019;13:9-16.

45. Bengtsson B, Leske MC, Hyman L, Heijl A, Early Manifest Glaucoma Trial Group. Fluctuation of intraocular pressure and glaucoma progression in the early manifest glaucoma trial. Ophthalmology. 2007;114(2):205209.

46. Medeiros FA, Weinreb RN, Zangwill LM, et al. Long-term intraocular pressure fluctuations and risk of conversion from ocular hypertension to glaucoma. Ophthalmology. 2008;115(6):934-940.

47. Caprioli J, Coleman AL. Intraocular pressure fluctuation a risk factor for visual field progression at low intraocular pressures in the advanced glaucoma intervention study. Ophthalmology. 2008;115(7):11231129.e3.

48. Tojo N, Hayashi A, Otsuka M. Correlation between 24-h continuous intraocular pressure measurement with a contact lens sensor and visual field progression. Graefes Arch Clin Exp Ophthalmol. 2020;258(1):175-182.

49. David R, Zangwill L, Briscoe D, Dagan M, Yagev R, Yassur Y. Diurnal intraocular pressure variations: an analysis of 690 diurnal curves. Br J Ophthalmol. 1992;76(5):280-283.

50. Nakakura S, Nomura Y, Ataka S, Shiraki K. Relation between office intraocular pressure and 24-hour intraocular pressure in patients with primary open-angle glaucoma treated with a combination of topical 
antiglaucoma eye drops. J Glaucoma. 2007;16(2):201-204.

51. Yamagami J, Araie M, Aihara M, Yamamoto S. Diurnal variation in intraocular pressure of normal-tension glaucoma eyes. Ophthalmology. 1993;100(5):643-650.

52. Loewen NA, Zhang $X$, Tan $\mathrm{O}$, et al. Combining measurements from three anatomical areas for glaucoma diagnosis using Fourier-domain optical coherence tomography. Br J Ophthalmol. 2015;99(9):1224-1229.

53. Mansouri K, Rao HL, Weinreb RN, ARGOS-02 Study Group. Short-Term and Long-Term Variability of Intraocular Pressure Measured with an Intraocular Telemetry Sensor in Patients with Glaucoma.

Ophthalmology. 2020;128(2):227-233.

54. Mansouri K, Gillmann K, Rao HL, Weinreb RN, ARGOS-2 Study Group. Weekly and seasonal changes of intraocular pressure measured with an implanted intraocular telemetry sensor. Br J Ophthalmol. 2020;105(3):387-391.

55. Cutolo CA, De Moraes CG, Liebmann JM, et al. The Effect of Therapeutic IOP-lowering Interventions on the 24-hour Ocular Dimensional Profile Recorded With a Sensing Contact Lens. J Glaucoma. 2019;28(3):252257.

56. Rüfer F, Gillmann K, Choritz L, Thieme H, Weinreb RN, Mansouri K. The Value of Intraocular Pressure Telemetry in Monitoring the Therapeutic Effect of Glaucoma Medications. J Glaucoma. 2020;29(6):e38-e40.

57. Liu JHK, Zhang X, Kripke DF, Weinreb RN. Twenty-four-hour intraocular pressure pattern associated with early glaucomatous changes. Invest Ophthalmol Vis Sci. 2003;44(4):1586-1590.

58. Lee YR, Kook MS, Joe SG, et al. Circadian (24-hour) pattern of intraocular pressure and visual field damage in eyes with normal-tension glaucoma. Invest Ophthalmol Vis Sci. 2012;53(2):881-887.

59. Saccà SC, Rolando M, Marletta A, Macrí A, Cerqueti P, Ciurlo G. Fluctuations of intraocular pressure during the day in open-angle glaucoma, normal-tension glaucoma and normal subjects. Ophthalmologica. 1998;212(2):115-119.

60. Joe SG, Choi J, Sung KR, Park SB, Kook MS. Twenty-four hour blood pressure pattern in patients with normal tension glaucoma in the habitual position. Korean J Ophthalmol. 2009;23(1):32-39.

61. Bonomi L, Marchini G, Marraffa M, Bernardi P, Morbio R, Varotto A. Vascular risk factors for primary open angle glaucoma: the Egna-Neumarkt Study. Ophthalmology. 2000;107(7):1287-1293.

62. Galassi F, Giambene B, Menchini U. Ocular perfusion pressure and retrobulbar haemodynamics in pseudoexfoliative glaucoma. Graefes Arch Clin Exp Ophthalmol. 2008;246(3):411-416.

63. Raman P, Suliman NB, Zahari M, Kook M, Ramli N. Low nocturnal diastolic ocular perfusion pressure as a risk factor for NTG progression: a 5-year prospective study. Eye . 2018;32(7):1183-1189.

64. Realini T, Weinreb RN, Wisniewski SR. Diurnal intraocular pressure patterns are not repeatable in the short term in healthy individuals. Ophthalmology. 2010;117(9):1700-1704.

65. Arora R, Bellamy H, Austin M. Applanation tonometry: a comparison of the Perkins handheld and Goldmann slit lamp-mounted methods. Clin Ophthalmol. 2014;8:605-610.

66. Baskett JS, Goen TM, Terry JE. A comparison of Perkins and Goldmann applanation tonometry. J Am Optom Assoc. 1986;57(11):832-834. 
67. Barkana Y, Gutfreund S. Measurement of the difference in intraocular pressure between the sitting and lying body positions in healthy subjects: direct comparison of the Icare Pro with the Goldmann applanation tonometer, Pneumatonometer and Tonopen XL. Clin Experiment Ophthalmol. 2014;42(7):608-614.

68. Liu JHK, Sit AJ, Weinreb RN. Variation of 24-hour intraocular pressure in healthy individuals: right eye versus left eye. Ophthalmology. 2005;112(10):1670-1675.

69. Hager H. Tagesdruckkurve und "Eintropfenkurve" also Grundlage fur die medikamentosa Einstellung des Glaukoms. Ber dtsch ophth Gel. 1957;60:318.

70. G-DRG System. Published September 18, 2018. Accessed June 13, 2021. https://reimbursement.institute/glossar/g-drg-system/

71. Dobromyslov AN, Panina NB. [Ambulatory care of patients with (surgically treated) glaucoma]. Oftalmol Zh. 1987;(8):505-506.

72. Beatty S, Kheterpal S, Eagling EM, O'Neill EC. Day-case trabeculectomies: Safety and efficacy. Acta Ophthalmol Scand. 1996;74(2):132-134. 
Tables

Table 1

Table 1: Demographics parameters of included patients.

\begin{tabular}{|c|c|c|c|c|}
\hline & $\begin{array}{l}\text { Males } \\
(n=88)\end{array}$ & $\begin{array}{l}\text { Females } \\
(n=137)\end{array}$ & p-value & Total \\
\hline age (years) & $72.8 \pm 12.6$ & $77.0 \pm 10.0$ & $0.006^{*}$ & $75.4 \pm 11.2$ \\
\hline $\begin{array}{c}\text { central corneal } \\
\text { thickness } \\
\text { (micrometers) }\end{array}$ & $534.8 \pm 38.3$ & $538.6 \pm 34.0$ & 0.43 & $536.3 \pm 35.7$ \\
\hline $\begin{array}{c}\text { average number of } \\
\text { drops }\end{array}$ & $2.2 \pm 1.6$ & $2.2 \pm 1.5$ & 1.00 & $2.2 \pm 1.5$ \\
\hline $\begin{array}{c}\text { average number of } \\
\text { surgeries }\end{array}$ & $0.6 \pm 0.7$ & $0.6 \pm 0.8$ & 0.77 & $0.6 \pm 0.7$ \\
\hline $\mathrm{T}_{\text {avg }}(\mathrm{mmHg})$ & $15.9 \pm 5.0$ & $14.7 \pm 3.1$ & 0.03 & $15.2 \pm 4.0$ \\
\hline $\mathrm{T}_{\max }(\mathrm{mmHg})$ & $20.3 \pm 6.9$ & $18.7 \pm 4.0$ & 0.03 & $19.3 \pm 5.4$ \\
\hline$I^{\prime O P} P_{\text {var }}(\mathrm{mmHg})$ & $7.4 \pm 4.9$ & $6.6 \pm 3.7$ & 0.17 & $6.9 \pm 4.2$ \\
\hline MOPP (mmHg) & $58.8 \pm 9.0$ & $59.3 \pm 8.8$ & 0.68 & $59.1 \pm 8.9$ \\
\hline
\end{tabular}




\section{Table 2}

Table 2. Comparison of sensitivity and specificity between progression as nominal variable and $\mathrm{IOP}_{\max }$ measurements using 15 and $22 \mathrm{mmHg}$ as cut-off values.

\begin{tabular}{|c|c|c|c|c|}
\hline cut-off value & parameter & 24h-IOP & OP-IOP & difference \\
\hline \multirow{2}{*}{$15 \mathrm{mmHg}$} & sensitivity & 0.69 & 0.63 & 0.06 \\
& specificity & 0.23 & 0.40 & -0.17 \\
\hline \multirow{2}{*}{$22 \mathrm{mmHg}$} & sensitivity & 0.07 & 0.06 & 0.01 \\
& specificity & 0.87 & 0.89 & -0.02 \\
\hline
\end{tabular}

OP-IOP = IOP measurements during out-patient hours (10 AM, 2 PM, 5 PM). 


\section{Table 3}

Table 3. Correlation coefficients for IOP and progression

\begin{tabular}{|c|c|c|c|c|c|}
\hline & $\mathrm{T}_{\text {avg }}$ & $T_{\max }$ & $T_{\min }$ & IOP $_{\text {var }}$ & MOPP \\
\hline $\mathrm{T}_{\text {avg }}$ & - & & & & \\
\hline$T_{\max }$ & $0.74^{*}$ & - & & & \\
\hline$T_{\min }$ & $0.87^{*}$ & $0.54^{*}$ & - & & \\
\hline $10 P_{v a r}$ & 0.11 & $0.64 *$ & $-0.21 *$ & - & \\
\hline MOPP & $-0.14^{*}$ & $-0.15^{*}$ & $-0.14^{*}$ & -0.025 & - \\
\hline G SL & -0.09 & -0.04 & 0.06 & -0.01 & -0.05 \\
\hline TS SL & -0.04 & -0.1 & -0.15 & $<-0.01$ & $-0.09 *$ \\
\hline T SL & -0.05 & -0.05 & 0.03 & -0.04 & -0.04 \\
\hline TI SL & -0.11 & -0.09 & -0.01 & -0.01 & -0.02 \\
\hline
\end{tabular}

Spectralis OCT parameters G SL = slope of global RNFL loss, TS SL = slope of temporal-superior RNFL loss, $\mathrm{T}$ SL = slope of temporal RNFL loss, $\mathrm{TI} \mathrm{SL}=$ slope of temporal-inferior RNFL loss, MOPP = mean ocular perfusion pressure, $*=$ significant at 0.05 


\section{Figures}

\section{Figure 1}

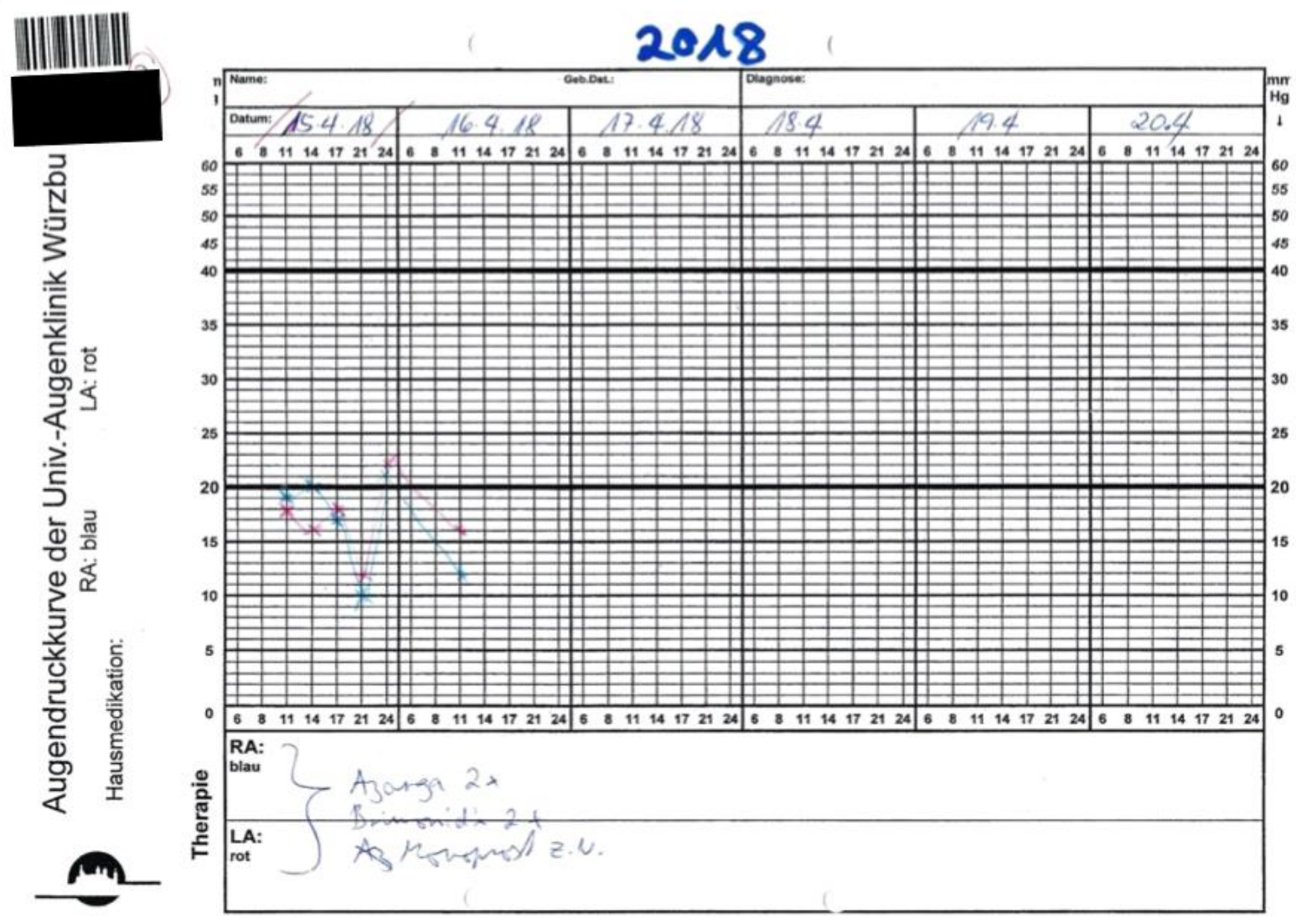

Figure 1. Example of an IOP chart used throughout the country of this study to this day. The time is displayed on a non-linear $x$-axis with uneven intervals and the IOP on a non-linear $y$-axis with a scale compressed above 40 $\mathrm{mmHg}$. The length of the $\mathrm{x}$-axis of this chart template indicates that IOP curves were sometimes obtained for six days. Blue= right eye, red= left eye. $A$ patient-identifying sticker is blacked out in the left upper corner. 


\section{Figure 2}
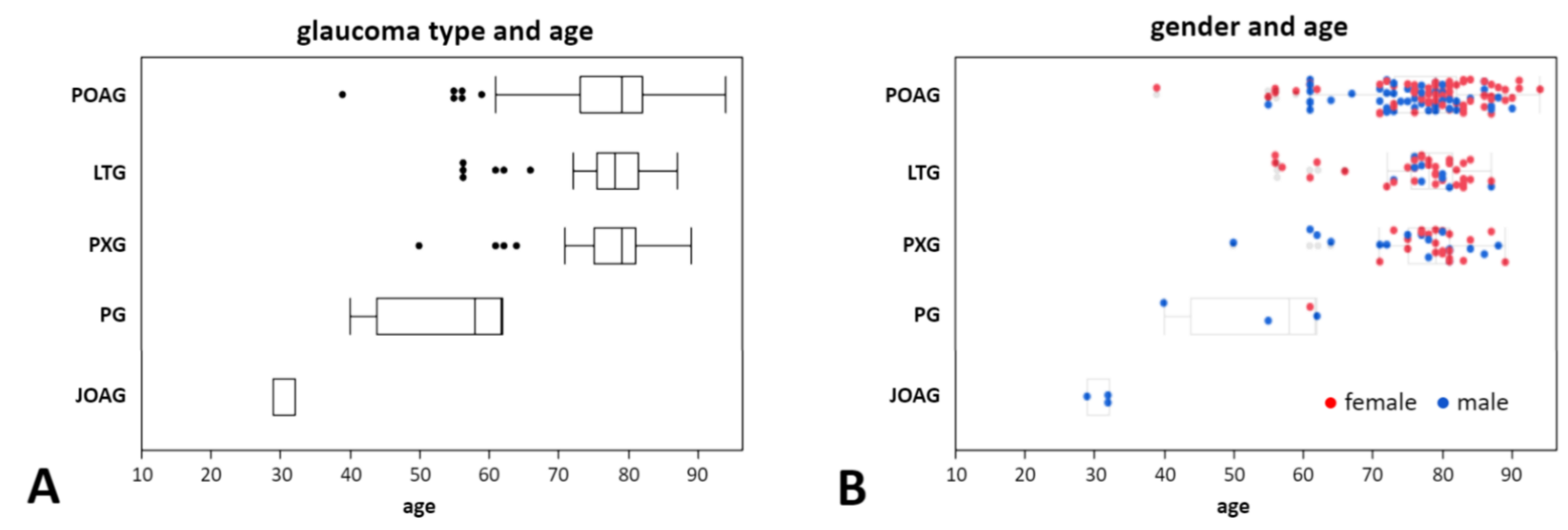

Figure 2: A) Glaucoma type and age distribution. POAG, LTG, PXG, and GS patients had similar averages, while PG were younger and JOAG were the youngest. B) Gender and age distribution. There were disproportionately more female LTG patients who were younger than male LTG patients. 


\section{Figure 3}

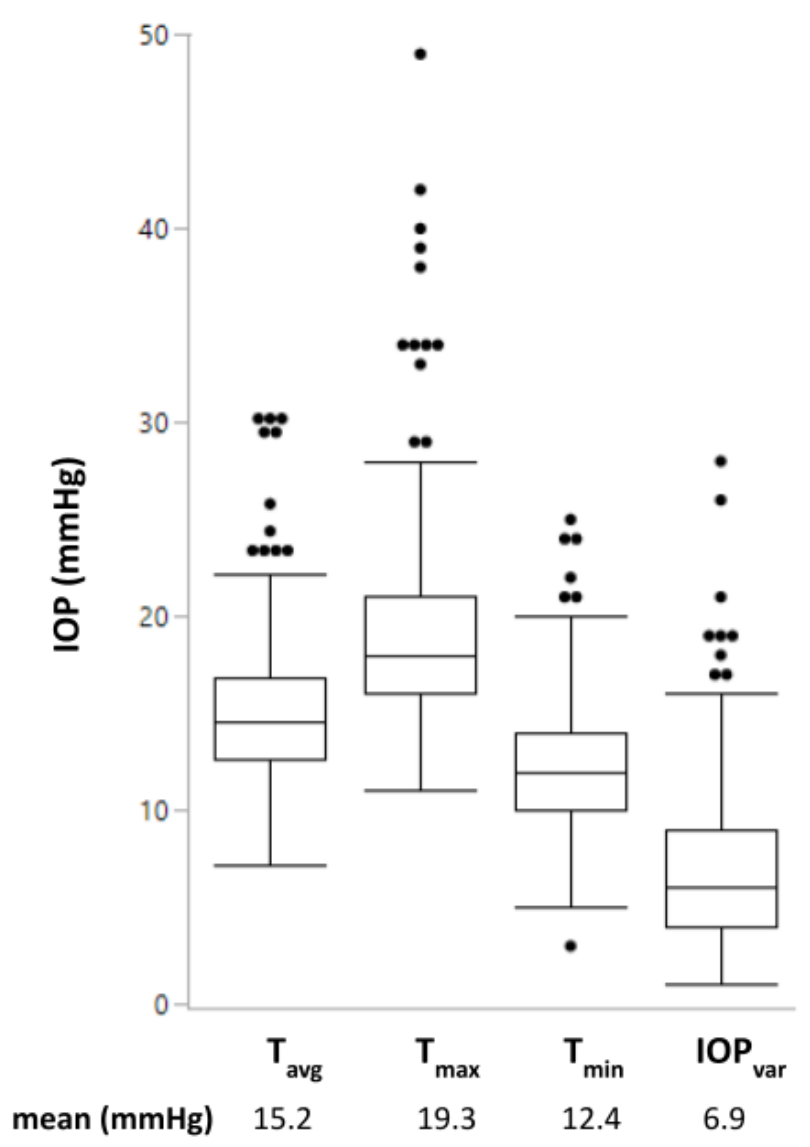

Figure 3: IOP average, maxima, minima, and variation. 


\section{Figure 4}
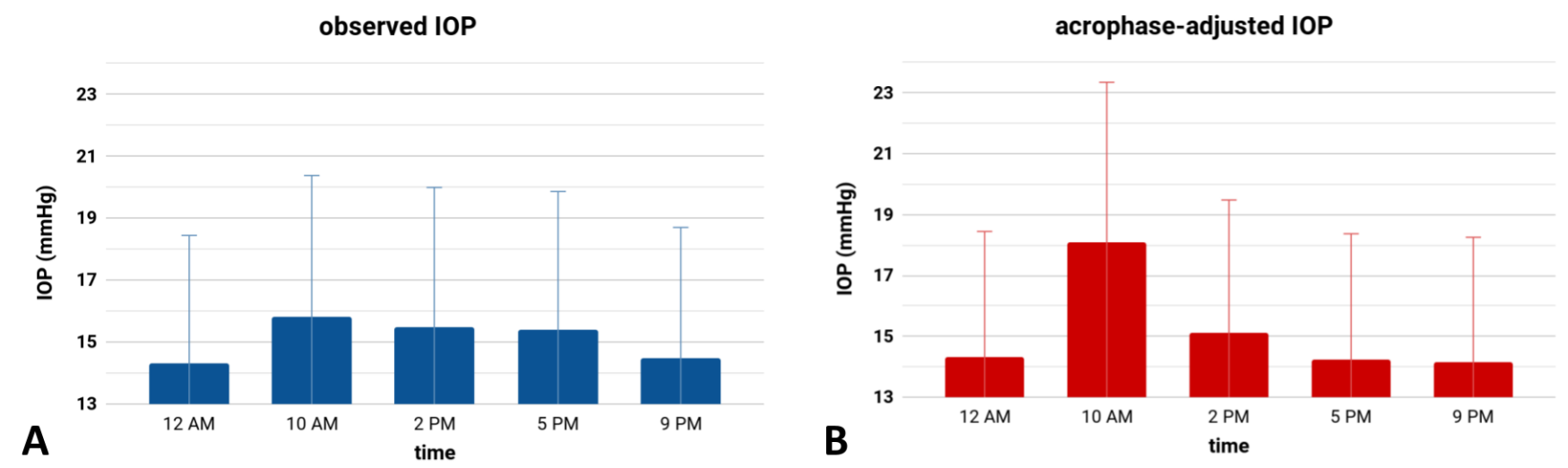

Figure 4: Nycthemeral (24-hour) IOPs as observed (A) and when arranged by estimated acrophases (B). 
Figure 5

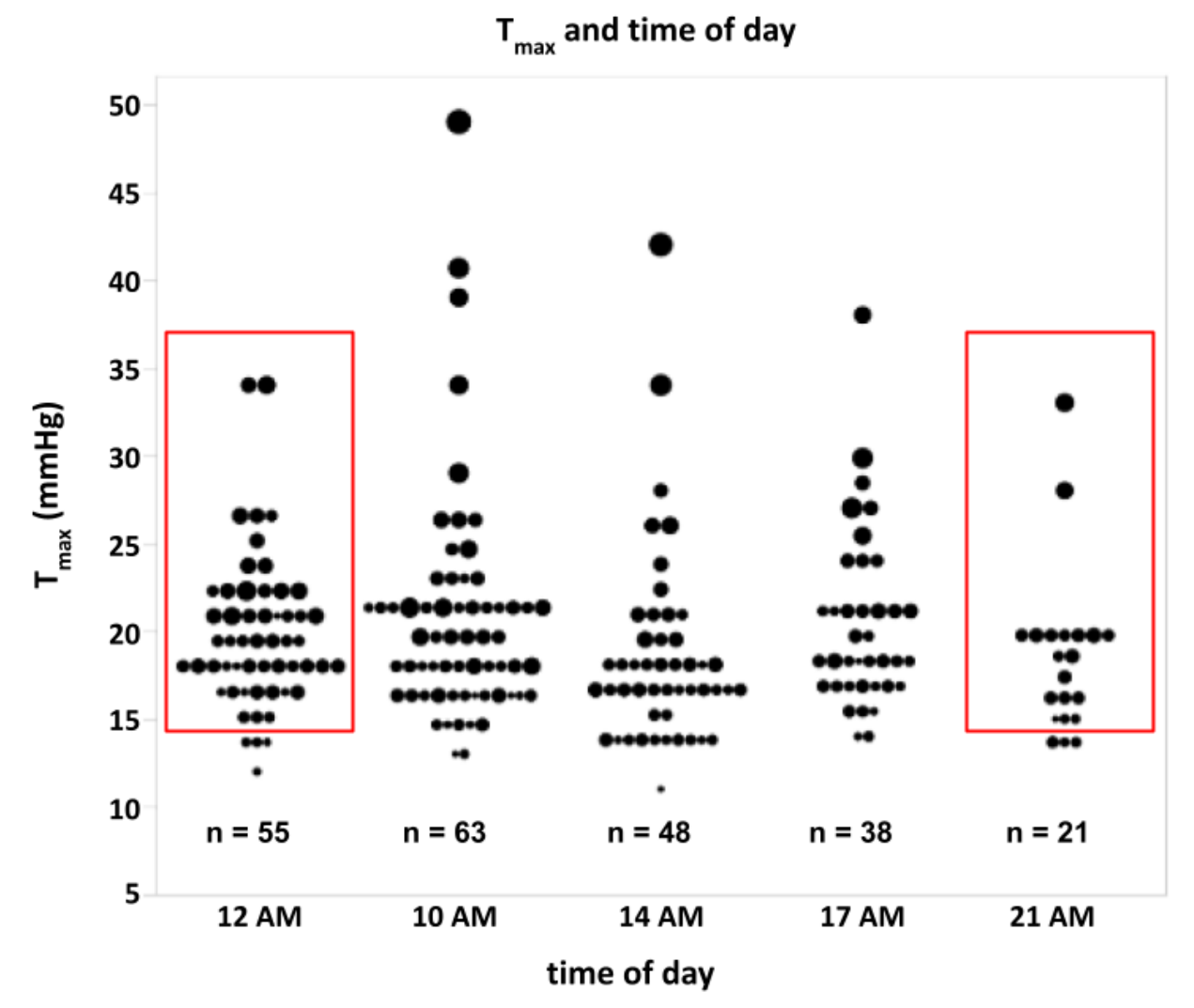

Figure 5: $T_{\max }$ and time of day at which $T_{\max }$ was reached. Each bubble represents the $T_{\max }$ of one patient during the $24 \mathrm{~h}$ IOP inpatient measurement. The bubble size indicates the amount of 24-hour IOP variation. Red boxes indicate $T_{\max }$ measurements above $15 \mathrm{mmHg}$ that would not be detected during typical outpatient office hours. 


\section{Figure 6}

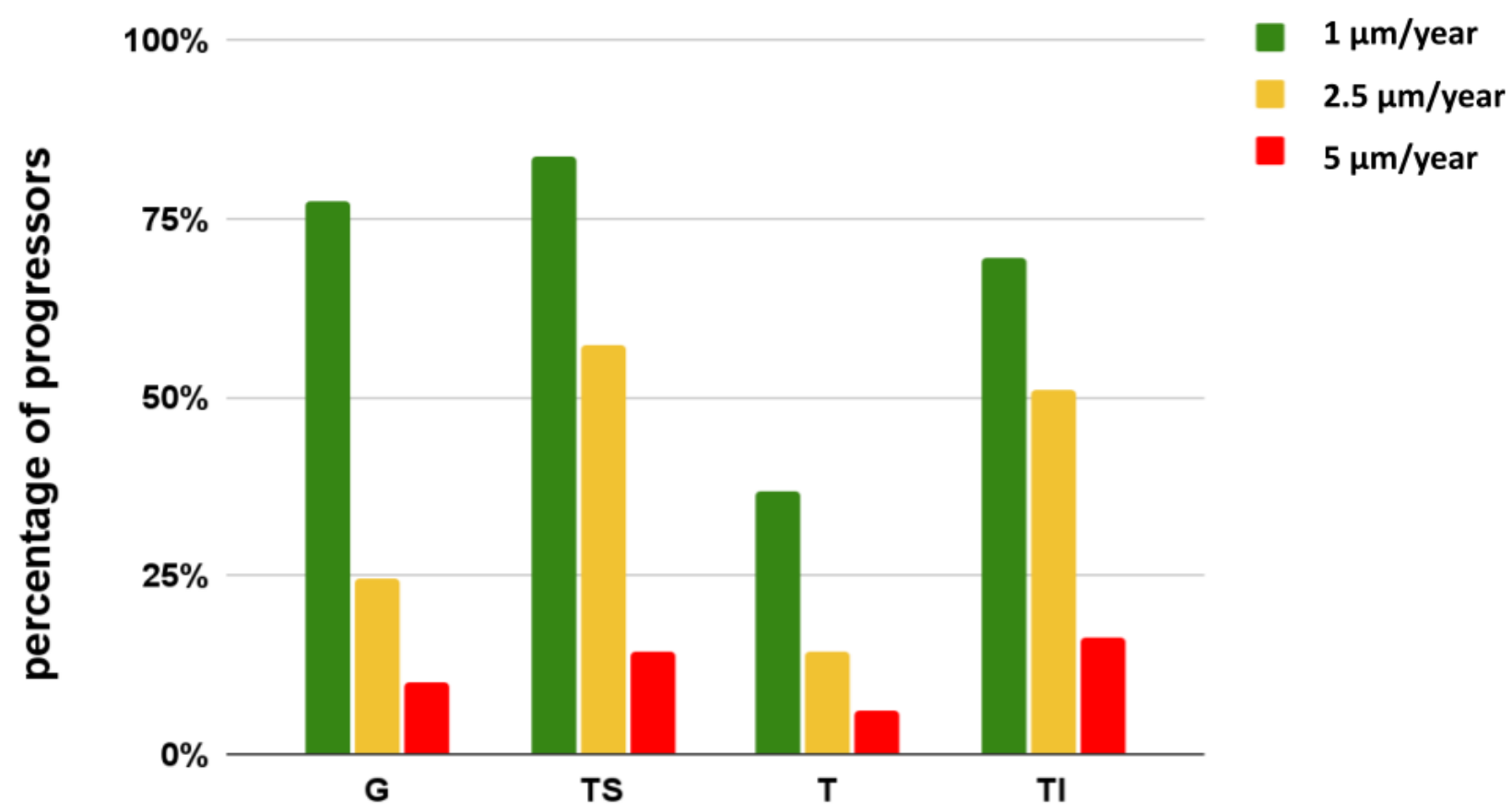

Figure 6: The percentage of progressors who had a retinal nerve fiber layer loss of at least 1 (green), 2.5 (yellow), and 5 (red) micrometers per year. G: global peripapillary region. TS: temporal-superior quadrant. T: temporal quadrant. TI: temporal-inferior quadrant. 


\section{Figure 7}

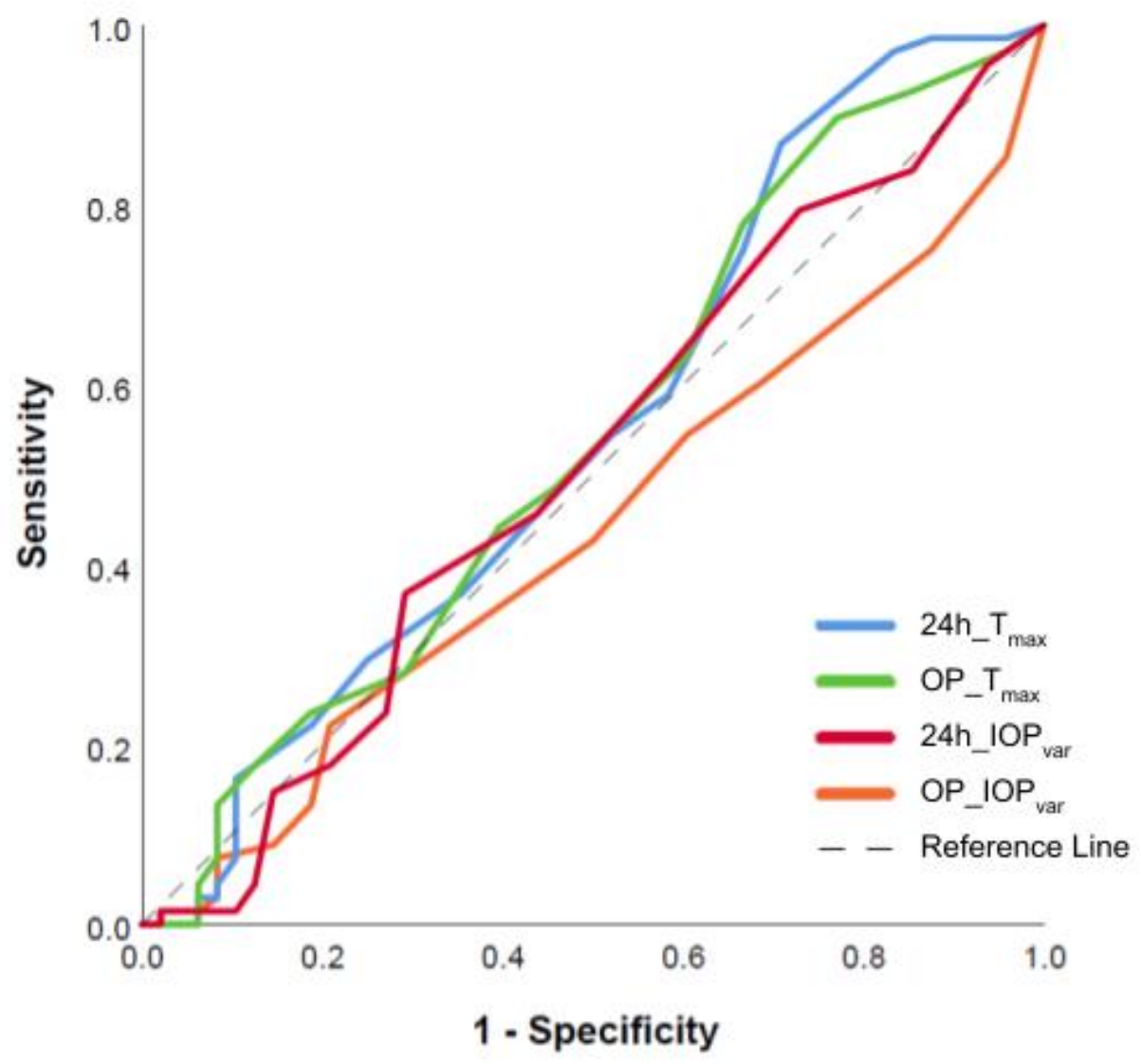

Figure 7. Receiver operating characteristic (ROC) curves comparing 24-hour and outpatient parameters of $T_{\max }$ and $\mathrm{IOP}_{\mathrm{var}}$ for disease progression. $\mathrm{IOP}_{\mathrm{var}}$ values of $<5 \mathrm{mmHg}$ were excluded from the analysis. This figure shows a very low predictive power of disease progression for all parameters. Well-performing tests have a hyperbolic ROC curve with sensitivity and specificity close to $90 \%$.

$24 \mathrm{~h}=$ nycthemeral measurements; $\mathrm{OP}=$ measurements during outpatient times. 